\title{
Nonlinear System Identification Using Neural Network
}

\author{
Muhammad Asif Arain ${ }^{1,2}$, Helon Vicente Hultmann Ayala ${ }^{1,2}$, \\ and Muhammad Adil Ansari ${ }^{3}$ \\ ${ }^{1}$ University of Genova, Italy \\ ${ }^{2}$ Warsaw University of Technology, Poland \\ a.arain@yahoo.com, helonayala@gmail.com \\ ${ }^{3}$ Quaid-e-Awam University of Engineering, Science \& Technology, Pakistan \\ maa17_84@yahoo.com
}

\begin{abstract}
Magneto-rheological damper is a nonlinear system. In this case study, system has been identified using Neural Network tool. Optimization between number of neurons in the hidden layer and number of epochs has been achieved and discussed by using multilayer perceptron Neural Network.
\end{abstract}

Keywords: Nonlinear systems, System identification, Magneto-rheological damper, Neural Networks.

\section{Introduction}

Magneto-rheological (MR) dampers are semi-active control devices to reduce vibrations of various dynamic structures. MR fluids, whose viscosities vary with input voltages/currents, are exploited in providing controllable damping forces. MR dampers were first introduced by Spencer to civil applications in mid- 1990s. In 2001, MR dampers were applied to the cable-stayed Dongting Lake Bridge in China and the National Museum of Emerging Science and Innovation Building in Japan, which are the world's first full-scale implementations in civil structures [1]. Modeling of MR dampers has received considerable attention [2-4]; however, these proposed models are often too complicated for practical usage. Recently, [5] proposed a so-called nonparametric model that has demonstrated two merits so far [6]:

1. The model can be numerically solved much faster than the existing parametric models;

2. The stability of an MR damper control system can be proved by adopting the nonparametric model. If currents/voltages of MR dampers are constants, the nonparametric model becomes a Hammerstein system depicted in Figure 1.

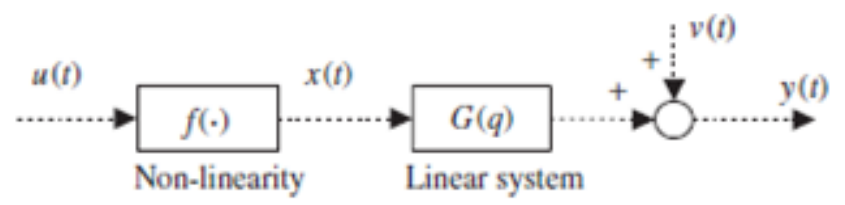

Fig. 1. A discrete-time Hammerstein system 
Here the input $u(t)$ and output $y(t)$ stand for the velocity and damping force, respectively. [5] suggested a first-order model for the linear system,

$$
G(q)=\frac{b_{1} q^{-1}}{1+a_{1} q^{-1}}
$$

and three candidate functions for the non-linearity,

$$
\begin{gathered}
f(u)=c_{1} \tanh \left(c_{0} u\right)+c_{2} \\
f(u)=c_{1} \operatorname{sgn}(u)\left[1-\exp \left(-c_{0}|u|\right)\right]+c_{2} \\
f(u)=\frac{\left(c_{0}+c_{1}\left|u-c_{3}\right|\right)^{c_{2}\left(u-c_{3}\right)}-\left(c_{0}+c_{1}\left|u-c_{3}\right|\right)^{-c_{2}\left(u-c_{3}\right)}}{c_{1} c_{2}\left(u-c_{3}\right)}+c_{0}^{-c_{2}\left(u-c_{3}\right)}
\end{gathered}
$$

Our objective is to design an identification experiment and estimate the output $\hat{y}(t)$ from the measured damping force $y(t)$ and velocity $u(t)$, using of neural networks black box models.

To study the behavior of such devices, a MR damper is fixed at one end to the ground and connected at the other end to a shaker table generating vibrations. The voltage of the damper is set to $1.25 \mathrm{~V}$. The damping force $f(t)$ is measured at the sampling interval of $0.005 \mathrm{~s}$. The displacement is sampled every $0.001 \mathrm{~s}$, which is then used to estimate the velocity $v(t)$ at the sampling period of $0.005 \mathrm{~s}$. The data used in this demo is provided by Dr. Akira Sano (Keio University, Japan) and Dr. Jiandong Wang (Peking University, China) who performed the experiments in a laboratory of Keio University. See [7] for a more detailed description of the experimental system and some related studies. We applied Neural Network for this nonlinear system identification because Neural Network stands out among other parameterized nonlinear models in function approximation properties and modeling nonlinear system dynamics [8]. And clearly, nonlinear system identification is more complex as compare to linear identification in a sense of computation and approximations. In section 2, Neural Network is designed with detailed description on it's I/Os (inputs, outputs), used data sets and training algorithm. In section 3, obtained results are presented in graphical and numerical forms. Section 4 discusses details about these obtained results. Finally, in section 5, case study conclusion is made.
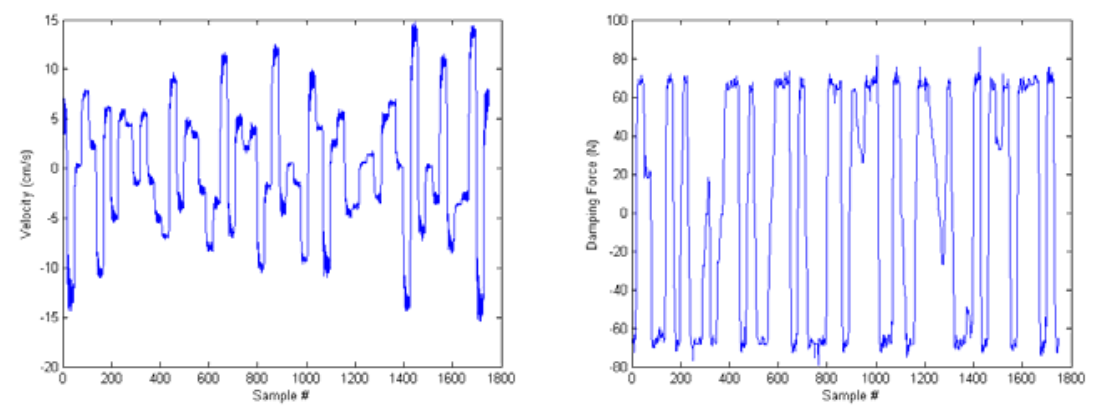

Fig. 2. System input (velocity) and output (damping force) 


\section{Neural Network Design}

Designing a Neural Network black-box model to perform the system identification of a nonlinear SISO (Single Input Single Output) system, namely (Magneto-rheological Damper); following design variables should be achieved.

\subsection{Input/output and Transfer Function}

\section{Neural Network input}

One delayed system output plus, one delayed system input and the actual input i.e. $y(k-1), u(k-1)$ and $u(k)$, are used as NN inputs. This results in a NARX model, similar to the Hammerstein model. As the results are satisfactory for the above cited NN inputs, the dynamic of the system could be captured with only one delay for each system input and output. There is no need to increase, then, the number of delays on the input and output.

The result for 50 epochs and 10 neurons and Levenberg-Marquadt backpropagation training procedure is presented below to justify this choice. For this case, a value of MSE (Mean Square Error) of 6.4180 is obtained.
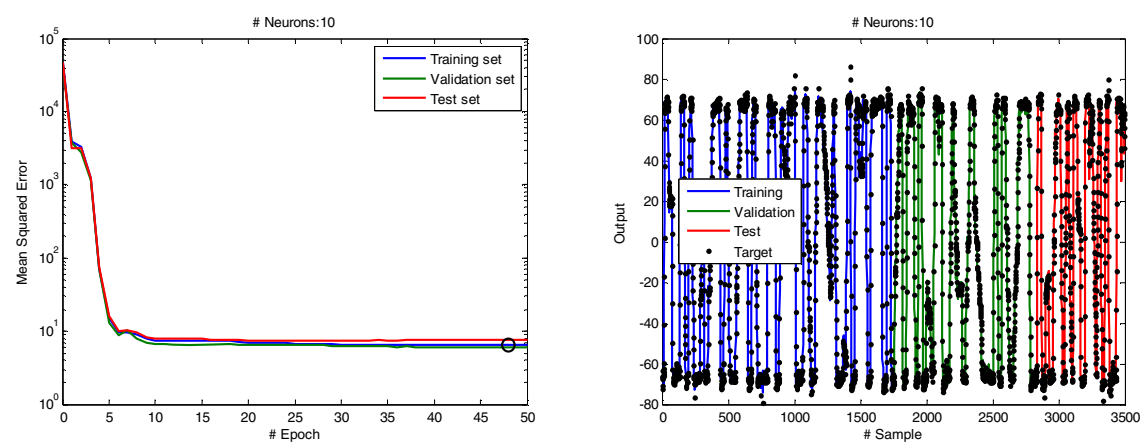

Fig. 3. Levenberg-Marquadt backpropagation training procedure

Neural Network output

For the neural network output it is used, obviously, the system output, that is $\hat{y}(k)$.

Neural Network transfer function

The NN transfer functions are chosen as hyperbolic tangent sigmoid (tansig) in hidden layer and pure linear (purelin) in output layer. 


\subsection{Data Sets}

The data set of nonlinear magneto-rheological dampers, provided by Dr. Akira Sano (Keio University, Japan) and Dr. Jiandong Wang (Peking University, China) contains 3499 input and output samples, each. The data is split into three sets;

i. One for training, containing first $50 \%$ of the amount of data.

ii. One for validating, containing second $30 \%$ (from $51 \%$ to $80 \%$ ) of the amount of data. This data set is used for checking the increase of error among the epochs - it is used mainly as stop criterion.

iii. One for testing, containing last $20 \%$ (from $81 \%$ to $100 \%$ ) of the amount of data. This data set does not influence the training procedure.

\section{The stop criteria}

The stop criterion of number of epochs for checking the increase of error on the validation data set is set as the number of epochs +1 , so the training procedure never stops because of that. In this study it is focused the effect of number of neurons and number of epochs on the result. Practically there are two data sets: one for training and another for testing, the last being constituted of the validation and test data sets.

\subsection{Training Algorithm}

The gradient descent backpropagation is tested, and it diverged for a set of number of neurons on the hidden layer. One hidden layer is used. An example for 10 neurons and 50 epochs is shown in Fig 4. Two training procedures are used. In first, number of epochs is fixed and numbers of neurons are varying. In second, number of neuron is fixed and numbers of epochs are varying.
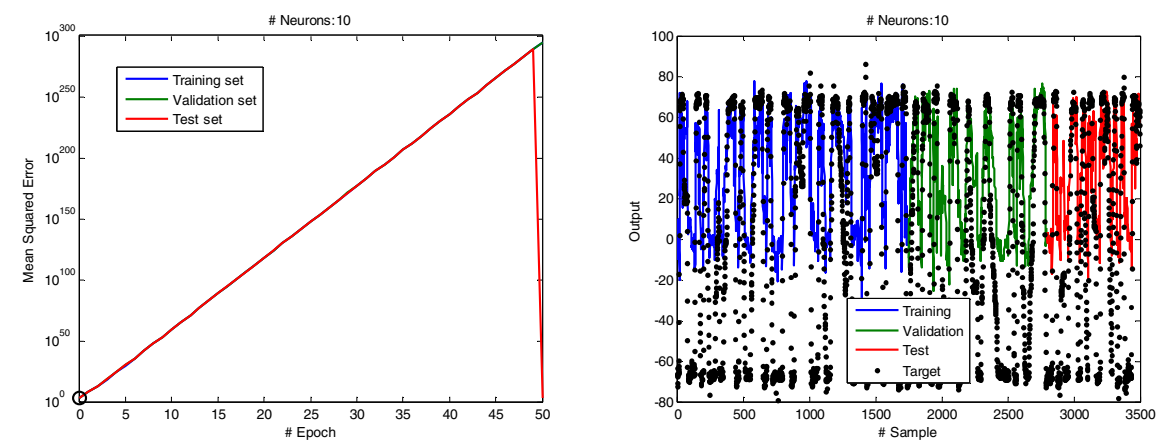

Fig. 4. Gradient Descent Backpropagation diverges 


\section{Varying neurons and fixed epochs}

Test the performance, for 50 epochs, of 2, 4, 6, 8, 10, 12 numbers of neurons in the hidden layer.

i. The results are shown in Table 1 and Figure 5.

ii. MSE values are calculated for training, validation and test datasets.

Fixed neurons and varying epochs

For 10 neurons in the hidden layer, change different number of epochs as 20, 100, $300,500,700$ and 1000 .

i. $\quad$ The results are shown in Table 2 and Figure 6.

ii. MSE values were calculated for training, validation and test datasets.

\section{Result criteria}

i. Calculate the MSE for all cases. The MSE represents the metric adopted for the training.

ii. Plot neural network output and desired output in the same graph (for comparing) for all cases.

iii. Plot neural network output error $(e(k)=y(k)-\hat{y}(k))$ for all cases.

iv. Analyze general results.

v. What is the influence of the number of neurons in a fixed number of epochs on the NN result?

vi. What is the influence of the number of epochs on the NN training performance?

\section{$3 \quad$ Results}

Using above training algorithm, obtained results are presented both in graphical and numerical forms. Fig. 5 shows graphical results obtained using different number of neurons and fixed epochs. Fig. 6 shows graphical results using fixed neurons and different number of epochs. In graphical results, MSE is plotted on logarithmic scale. Instead of using error only, MSE (mean square error) variable was used to show a clear identification of error trend in training, validation and testing sets with respect to number of epochs, this comparison is shown in Fig.7. In numerical results, MSE in training, validation and testing sets against variable neurons and epochs is presented in Table 1 and Table 2 respectively.

Graphical Results 

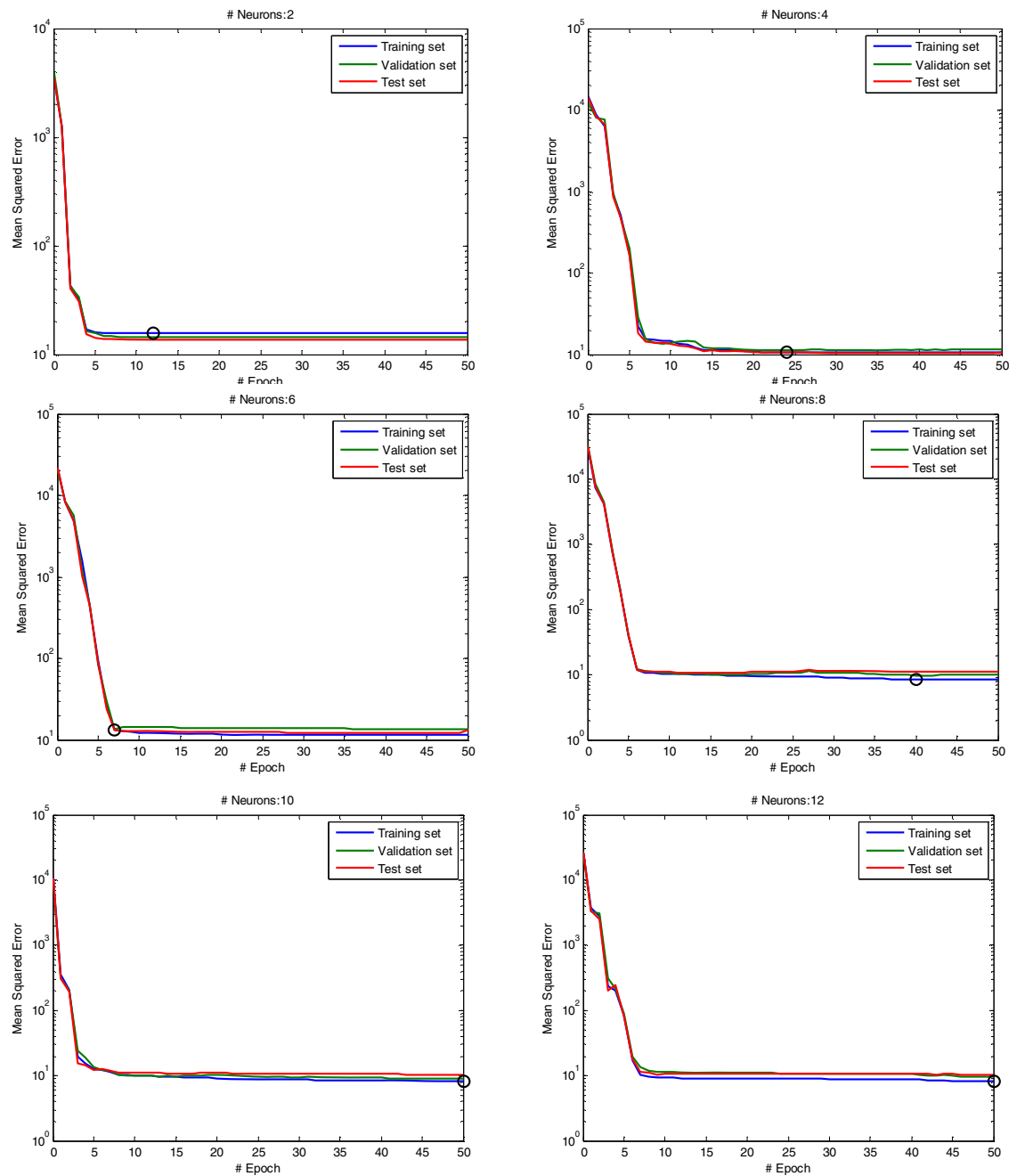

Fig. 5. Results for 50 (fixed) epochs and 2, 4, 6, 8, 10 and 12 neurons in the hidden layer 

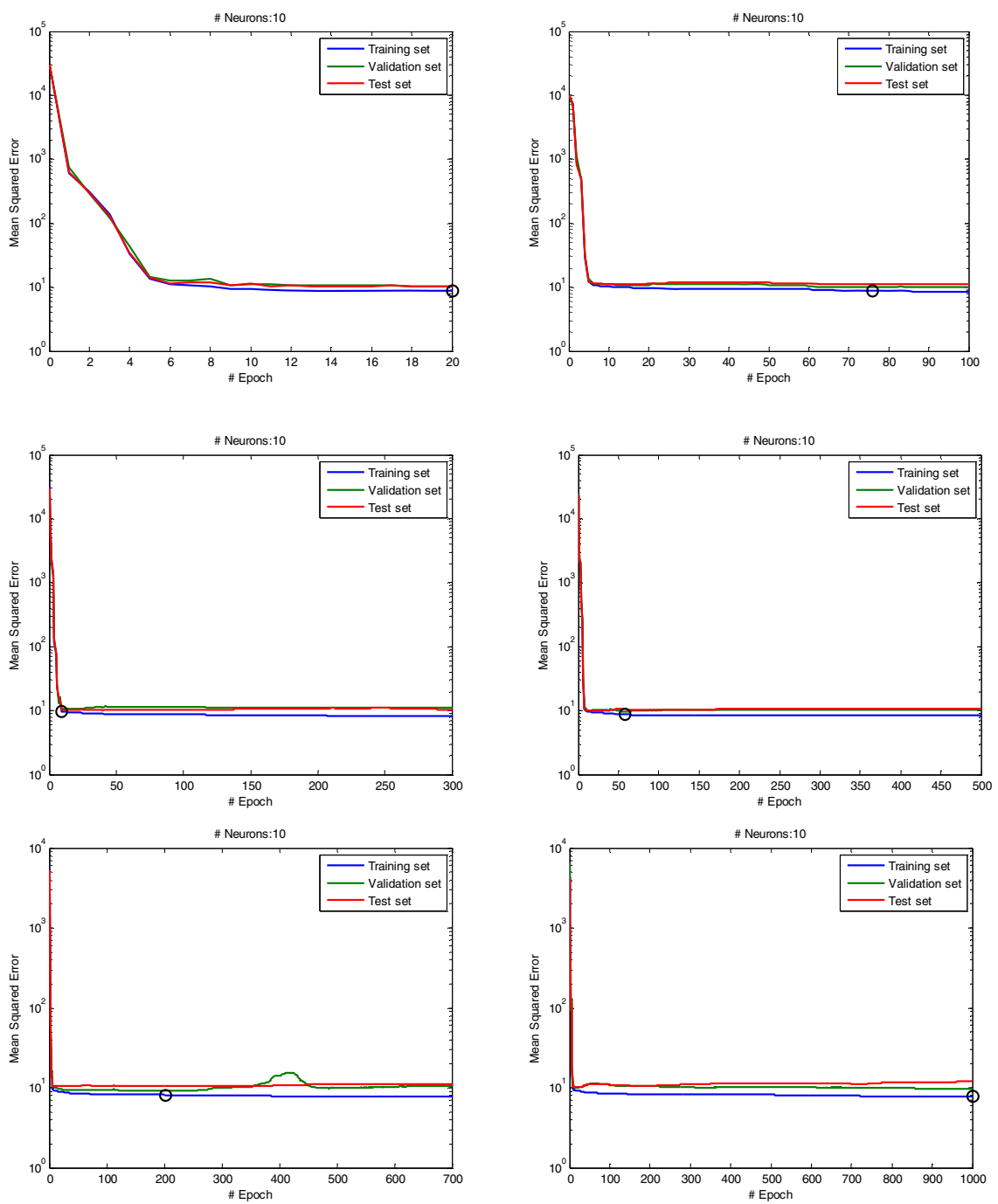

Fig. 6. Results for $20,100,300,500,700$ and 1000 epochs and 10 (fixed) neurons in the hidden layer 

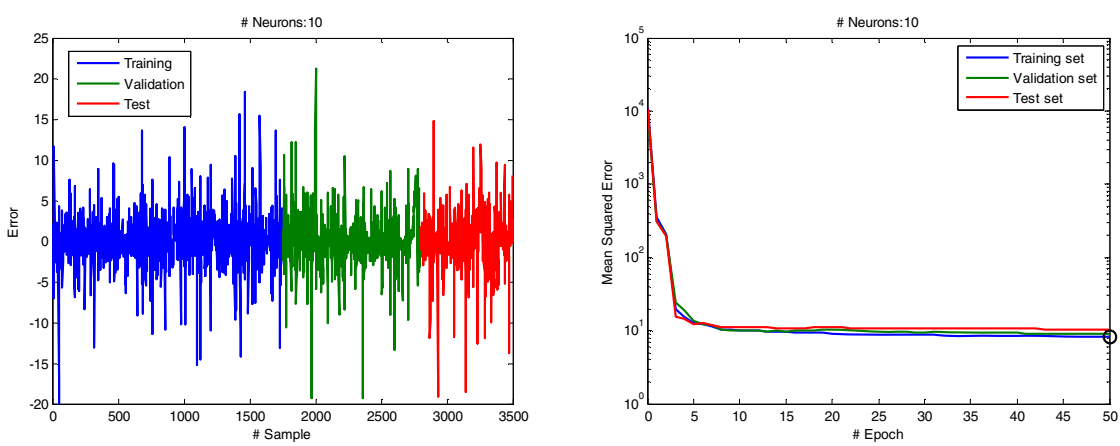

Fig. 7. Representation of error and MSE for 10 neurons in hidden layer

Numerical Results

Table 1. Results for different number of neurons and 70 epochs

\begin{tabular}{cccc}
\hline Neurons & MSE - Train & MSE - Validation & MSE - Test \\
\hline 2 & 15.6728707486869 & 14.4948575906152 & 13.6752183182602 \\
4 & 10.7218539702842 & 11.3195658194943 & 10.6840535069316 \\
6 & 13.3840846836663 & 13.6716607191865 & 13.1696042197544 \\
\hline 10 & 8.40574921395610 & 9.84068816503996 & 11.0299877969621 \\
12 & 8.29385308661188 & 9.06040436519353 & 10.4993626210124 \\
\hline
\end{tabular}

Table 2. Results for different number of epochs and 10 neurons

\begin{tabular}{cccc}
\hline Epochs & MSE - Train & MSE - Validation & MSE - Test \\
\hline 20 & 8.46213141484072 & 9.28610314243466 & 10.5389069957705 \\
100 & 8.68527655115772 & 9.55504632661187 & 10.6903292802750 \\
300 & 8.37896217859245 & 8.80832992981236 & 10.2434588260025 \\
\hline 500 & 8.27327467204573 & 9.05391102870505 & 10.4767184896312 \\
700 & 8.15121872108450 & 9.82165043634970 & 10.4437982077475 \\
1000 & 7.90768469589262 & 8.99822232524322 & 10.7080085654322 \\
\hline
\end{tabular}




\section{Discussions}

The results shown are trying to expose the influence of the number of epochs and number of neurons issue in NN design. It can be noted, from Figure 5 and Table 1 that the number of neurons has a maximal value for improving the accuracy of the NN. From Table 1, one can say that the best value for the numbers of the NN hidden layer neurons is 10; with 12 neurons the error for the validation increases, though the test data error is diminished. This fact shows that the NN starts to represent over-fitting of the data with 12 neurons.

From Figure 6, it can be seen that there is also a maximum number of epochs that improve the NN results accuracy. Figure 6 shows that at epoch 700 the validation error starts to grow, what also can be seen as over-fitting of data. Moreover, it may also be noted that there is a number of epochs that the NN stops to improve the training data set result; around 70 epochs in the case studied in this work. The backpropagation algorithm stays at local minima (it may also be global) in this point. One may use several stop criteria for the backpropagation algorithm in order to avoid this drawback of limiting only the number of epochs.

General results show that the proposed designed NN can be a powerful tool to perform the systems identification with complex and nonlinear behavior. It is possible to affirm that the use of Levenberg-Marquadt backpropagation for training multilayer perceptrons has given accurate results, as shown by numerical expositions in Table 1 and 2 .

\section{Conclusion}

This case study presented an application of multilayer perceptron Neural Networks to perform the nonlinear system identification having its parameters defined by Levenberg-Marquadt Backpropagation training procedure. Such training procedure is chosen because the standard Steepest Descent Backpropagation does not converge for several sets of configurations. It is attempted, with this configuration, to build a blackbox model that could represent the system. The tested case study, the magnetorheological damper, is a nonlinear SISO system. For obtaining the exposed results, all methods are described and put under context.

Finally, the obtained results are considered satisfactory, showing that the present methodology can achieve the identification of the analyzed nonlinear system. The results could be observed on graphs and tables, where the MSE is presented in training, validation and test phases. The proposed methodology proved that it can be tested with systems having different characteristics, such as chaotic systems and controller design using neural network models. Future work could aim the adaptation of different optimization techniques to perform the $\mathrm{NN}$ training procedure in order to guarantee accuracy and generalization capability.

Acknowledgement. Authors are thankful to Prof. Lotfi Romdhane, Ecole Nationale d'Ingénieurs de Sousse, Tunisia for his guidelines. 


\section{References}

1. Cho, S.W., Jung, H.J., Lee, J.H., Lee, I.W.: Smart Passive System Based on MR Damper. In: JSSI 10th Anniversary Symposium on Performance of Response Controlled Buildings, Yokohama, Japan

2. Spencer, B.F., Dyke, S.J., Sain, M.K., Carlson, J.D.: Phenomenological Model of a Magneto-rheological Damper. ASCE Journal of the Engineering Mechanics 123, 230-238

3. Choi, S.B., Lee, S.K.: A Hysteresis Model for the Field-dependent Damping Force of a Magnetorheological Damper. Journal on Sound and Vibration 245, 375-383

4. Yang, G.: Large-Scale Magnetorheologoical Fluid Damper for Validation Mitigation: Modeling, Testing and Control. The University of Notre Dame, Indiana

5. Song, X., Ahmadian, M., Southward, S.C.: Modeling Magnetorheological Dampers with Application of Nonparametric Approach. Journal of Intelligence Material Systems and Structures 16, 421-432

6. Song, X., Ahmadian, M., Southward, S.C., Miller, L.R.: An Adaptive Semiactive Control Algorithm for Magneto-rheological Suspension Systems. Journal of Vibration and Acoustics $127,493-502$

7. Wang, J., Sano, A., Chen, T., Huang, B.: Identification of Hammerstein systems without explicit parameterization of nonlinearity. International Journal of Control

8. Subudhi, B., Jena, D.: Nonlinear System Identification of a Twin Rotor MIMO System. In: IEEE TENCON (2009) 